\title{
An Appreciation of Jim Pawley
}

William Bement

University of Wisconsin-Madison, Madison, Wisconsin, United States

Jim Pawley was my colleague in the Zoology Department at the University of Wisconsin-Madison for many years. His passion for and expertise in microscopy had an enormous impact on my research program and that of the others in our department.

After being a key part of the Integrated Microscopy Resource (IMR) at the University of WisconsinMadison (UW-Madison), Jim became a faculty member in the Department of Zoology (now the Department of Integrative Biology) at UW-Madison and set his lab up in the Zoology Research Building (now the Integrative Biology Research Building). Jim made it his mission to bring confocal microscopy to the Zoology Research Building and, accordingly, developed an instrumentation proposal for the National Science Foundation based on the development of a novel detector for the confocal system. He very graciously offered several other Zoology Research Building denizens, myself included, to serve as co-PIs on the proposal. The proposal was funded and Jim used the resources it provided to purchase a Biorad 1024 Laser Scanning Confocal Microscope. He set up a system whereby the instrument would be used part time to help him develop and vet the detector, but then used for the rest of the time for the acquisition of data on biological samples. he also arranged to teach a class to all of the faculty, postdocs and graduate students in the Zoology Research Building who were interested in learning about confocal microscopy.

I, my postdoc, and three of my graduate students immediately signed up for the course and began learning how to use the 1024. Prior to that time, we had used another Biorad instrument in the IMR for the analysis of fixed samples derived from cell free Xenopus (clawed frog) egg extracts. But thanks to Jim's tutelage, we made the happy discovery that not only was confocal technology useful for the high contrast samples provided by cell free egg extracts, it also worked extraordinarily well for living Xenopus oocytes, eggs and embryos. This finding ran contrary to the wisdom that prevailed at the time, which was that the Xenopus system was good for biochemistry and classic developmental biology, but rotten for any kind of live imaging. This conviction was based on the utter failure of standard epifluorescence to work with the yolky, heavily pigmented Xenopus eggs and embryos and was so heavily ingrained in the research community that researchers were at the time moving away from the Xenopus system to other models such as C. elegans and Zebrafish, which were considered to be far superior to Xenopus because of their translucence.

It is no exaggeration to say that Jim and his 1024 completely transformed our research program. We began to use confocal microscopy not simply as a tool of confirmation, but as a tool of discovery. Among other things, we learned that damage to the oocytes elicits a rapid signaling and cytoskeletal response in which the site of damage becomes surrounded by a ring of actin filaments and myosin- 2 that close over the wound; that exocytosis of egg secretory granules is accompanied by formation and compression of an actin filament "coat" around the secretory granules; that a novel microtubule-binding myosin (myosin-10) is essential for mitotic spindle assembly and dynamics; and that cytokinesis is triggered by formation of a stripe of activation of the small GTPase Rho. 


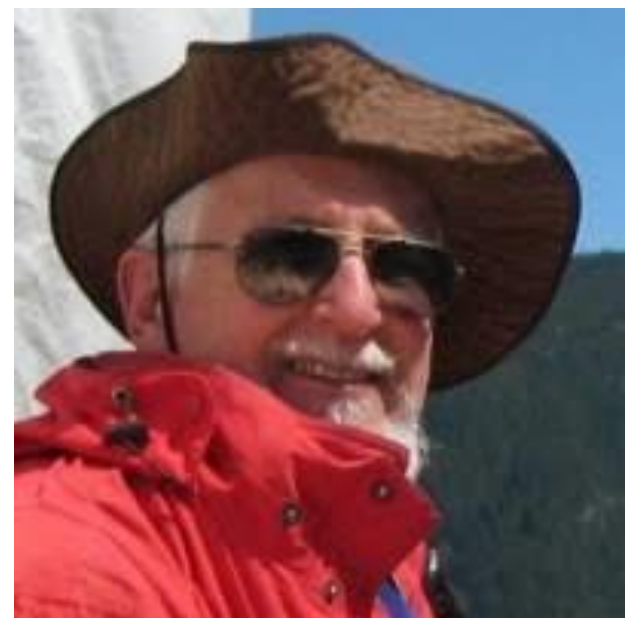

Figure 1. Jim Pawley

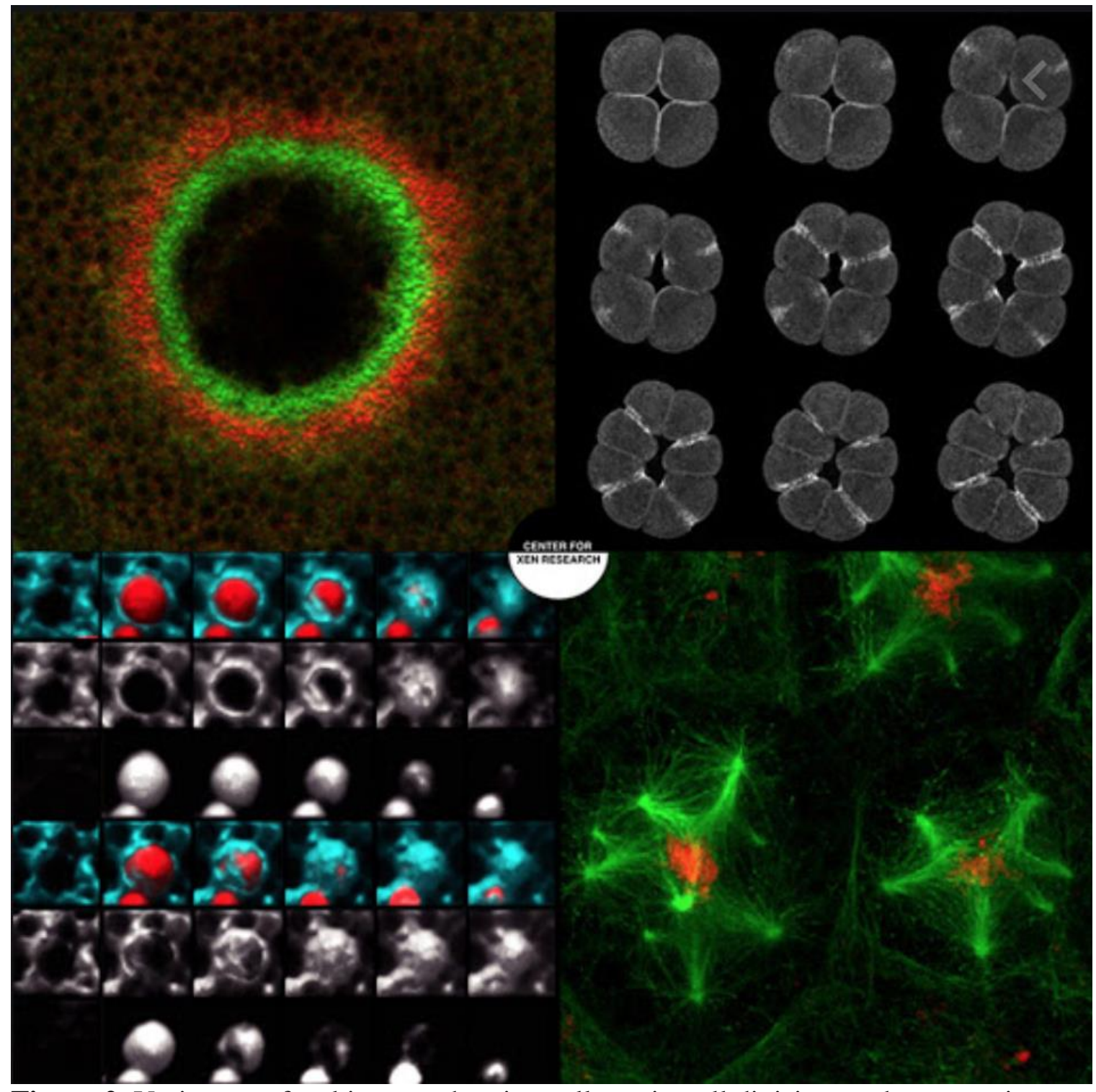

Figure 2. Various confocal images showing cell repair, cell division, and exocytosis. 